\title{
ARTICLE
}

\section{Radiation shielding experiments for composites based on pb-shot and boride}

\author{
Jun-ichi Hori $^{\mathrm{a}^{*}}$, Hiroshi Yashima ${ }^{\mathrm{a}}$, Keiichi Miyamoto ${ }^{\mathrm{b}}$, Shigeaki Okuda ${ }^{\mathrm{b}}$, Masanobu Ishihara ${ }^{\mathrm{b}}$, Shigeru Kito ${ }^{\mathrm{c}}$ and \\ Mutsumi Atarashic \\ ${ }^{a}$ Research Reactor Institute, Kyoto University, Asashiro-Nishi, Kumatori-cho, Sennan-gun, Osaka 590-0494, Japan; ${ }^{b}$ Shinsho Pb \\ industrial Co. LTD., Nonaka-Kita, Yodogawa-ku, Osaka, 532-0034, Japan; ${ }^{c}$ Fukuda Metal Foils \& Powder Co. LTD, \\ Nakatomi-cho, Yamashina-ku, Kyoto, 607-8305, Japan
}

\begin{abstract}
We have developed a new type of shielding material called as "Double Shield" which consists of Pb-shot 0.5 $\mathrm{mm}$ in diameter and boride for shielding both neutron and gamma rays effectively. The transmission neutron measurements were performed with the neutron time-of-flight technique using a $\mathrm{BF}_{3}$ detector and the neutron attenuation rate of the new product was compared with those of other shielding materials for each energy region. Moreover, the slab attenuation experiments were performed up to $30 \mathrm{~cm}$ for the new product, $\mathrm{Pb}$ and polyethylene mixed with boron oxide by measuring the leakage neutron and gamma-ray dose rates behind the materials. Based on the results of those experiments, it was verified that the new product has a higher shielding performance with respect to the total amount of neutron and gamma-ray dose rates.
\end{abstract}

Keywords: shielding material; Pb-shot; boride; transmission neutron; KURRI-Linac; Time-of-flight measurement; leakage neutron and gamma rays dose rate

\section{Introduction}

Radiation shielding material is an important component in nuclear facilities such as nuclear plants and accelerator facilities. Particularly it is an important issue to minimize the healthy tissue dose rate from all radiations such as neutron, primary and secondary gamma rays in the Boron Neutron Capture Therapy (BNCT) [1]. As the space of radiation shielding is restricted in the case of $\mathrm{BNCT}$, shielding materials with a high attenuation of both neutron and gamma rays are required. The contamination of absorbed dose by fast-neutron and gamma-ray components under the free-air condition was shown in Ref. [2]. The ratios of the gamma-ray dose to the fast-neutron dose were reported as about 26 and $13 \%$ in the cases of mixed radiation fields for BNCT with reactor-based and accelerator-based neutron sources, respectively. Therefore, it is important to develop the appropriate shielding material for the mixed radiation fields where the gamma-ray dose occupies more than $10 \%$ of the total dose. Moreover, the flexibility of material shape is also required for the shielding leakage radiations by streaming.

In this work, a new type of shielding material called as "Double Shield" which consists of $\mathrm{Pb}$-shot $0.5 \mathrm{~mm}$ in diameter and boride $\left(\mathrm{H}_{3} \mathrm{BO}_{3}\right)$ has been developed. To glue the particles of $\mathrm{Pb}$-shot and boride, $\mathrm{C}_{6} \mathrm{H}_{10} \mathrm{O}_{5}$ is mixed with the composite. The hydrogen content of the

*Corresponding author. Email: hori@ rri.kyoto-u.ac.jp material is $3.7 \times 10^{22} / \mathrm{cm}^{3}$. Inelastic scattering by lead and elastic scattering by hydrogen are effectively slowing down the velocity of fast neutron and ${ }^{10} \mathrm{~B}$ can reduce thermal neutrons as an absorber. The primary gamma rays from the neutron source and the secondary ones emitted from the neutron capture reactions in the shielding materials are attenuated by $\mathrm{Pb}$. Therefore, the material contains elements of lead and boride is expected to have high-performance shielding properties in the neutrons and gamma-rays mixed radiation fields. The specific gravity is 4.1 which is equivalents to twice of that of concrete. The construction cost is not much expensive compared with polyethylene and lead blocks. The "Double Shield" also has a flexibility of shapes such as board, brink and sealant.

We have carried out the transmission neutron and the slab attenuation measurements by using the $46-\mathrm{MeV}$ electron linear accelerator at Research Reactor Institute, Kyoto University (KURRI-Linac) as a photo-neutron source [3]. The neutron and gamma-rays shielding performance of the new shielding material was obtained

\section{Experiment}

\subsection{Transmission neutron measurement}

The experimental arrangement is shown in Figure 1. Pulsed fast neutrons were produced in a water-cooled photo-neutron target [3], which was $5 \mathrm{~cm}$ in diameter and $6.1 \mathrm{~cm}$ long and composed of 12 sheets of Ta plates 


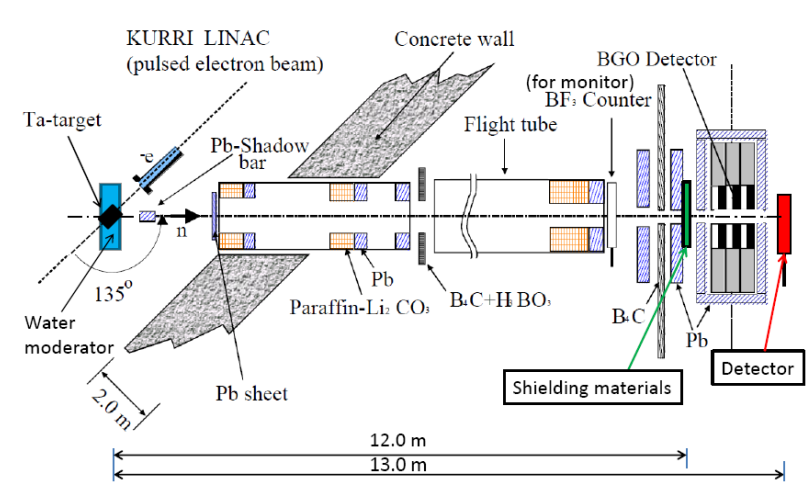

Figure 1. Experimental arrangement for the transmission neutron TOF and the slab attenuation measurement with the KURRI-Linac

with the total thickness of $29 \mathrm{~mm}$. The fast neutrons were moderated in an octagonal water tank, $30 \mathrm{~cm}$ in diameter and $10 \mathrm{~cm}$ thick, placed besides the target. The intense bremsstrahlung was also produced at the Ta target. Consequently, the moderated neutrons and intense gamma-rays mixed radiation fields were provided in the source.

The measurements of transmission neutrons for the shielding material were performed with the neutron time-of-flight (TOF) technique under the following Linac conditions: the electron energy, about $30 \mathrm{MeV}$; pulse repetition rate, $50 \mathrm{~Hz}$; pulse width, $100 \mathrm{~ns}$; and average electron current, about $17 \mu \mathrm{A}$. The flight path used in the experiment is in the direction of $135^{\circ}$ to the electron beam. The neutron beam was collimated to 2 $\mathrm{cm}$ in diameter with the collimation system composed of $\mathrm{B}_{4} \mathrm{C}, \mathrm{Li}_{2} \mathrm{CO}_{3}$ and $\mathrm{Pb}$ materials. A sample was placed at a distance of $12.0 \mathrm{~m}$ from the neutron source and the transmission neutrons were measured with a $\mathrm{BF}_{3}$ detector placed at a distance of $13.0 \mathrm{~m}$ from the neutron source. Blocks $(10 \mathrm{~cm}$ wide, $20 \mathrm{~cm}$ height and $5 \mathrm{~cm}$ thick) of the Double Shield, $\mathrm{Pb}$ and polyethylene mixed with $10 \%$ boron oxide were used as samples. The element content of Double Shield is shown in Table 1. The output signals from the detector and the trigger signals from the accelerator were stored with the list mode using the Yokogawa's WE7562 multi channel analyzer. The transmission neutron TOF spectra were obtained by gating on the pulse-height region for the ${ }^{10} \mathrm{~B}(\mathrm{n}, \alpha)$ reaction.

Table 1. Element content of the Double Shield

\begin{tabular}{cc}
\hline Element & $\begin{array}{c}\text { Number of density } \\
\times 10^{22}\left(/ \mathrm{cm}^{3}\right)\end{array}$ \\
\hline $\mathrm{Pb}$ & 0.814 \\
$\mathrm{~B}$ & 0.546 \\
$\mathrm{O}$ & 4.10 \\
$\mathrm{H}$ & 3.69 \\
$\mathrm{C}$ & 0.150 \\
\hline
\end{tabular}

\subsection{Slab attenuation measurement}

The leakage neutron and gamma-ray dose rates were measured behind the materials, respectively. A neutron remcounter (ALOKA TPS-451C) was used for the measurement of neutron dose equivalent. An ionization chamber (ALOKA ICS-313) was used for the measurement of gamma-ray dose equivalent. The positions of sample and detector were same as the transmission neutron measurement. Thickness of shielding material was increased by $5 \mathrm{~cm}$ up to $30 \mathrm{~cm}$.

\section{Calculation}

In order to estimate the neutron shielding effect due to the elements included in Double Shield, calculations with a Monte Carlo code MCNP-4C [4] were performed. A simplified model was used: a neutron source is located at the center of a sphere. In the sphere, we set two material regions with a thickness of $10 \mathrm{~cm}$ as shown in Figure 2. Those regions were filled with the shielding materials, respectively. The radius of the outer sphere is $150 \mathrm{~cm}$ and the neutron flux was estimated on the outer surface of the sphere. A Maxwell fission spectrum was used as a source. We performed three kinds of calculations as shown in Table 2. The densities of $\mathrm{Pb}$ and $\mathrm{H}_{3} \mathrm{BO}_{3}$ used in the calculation- 2 and 3 were adjusted as the effective thickness of each nucleus was equal to that of Double Shield in the calculation- 1 .

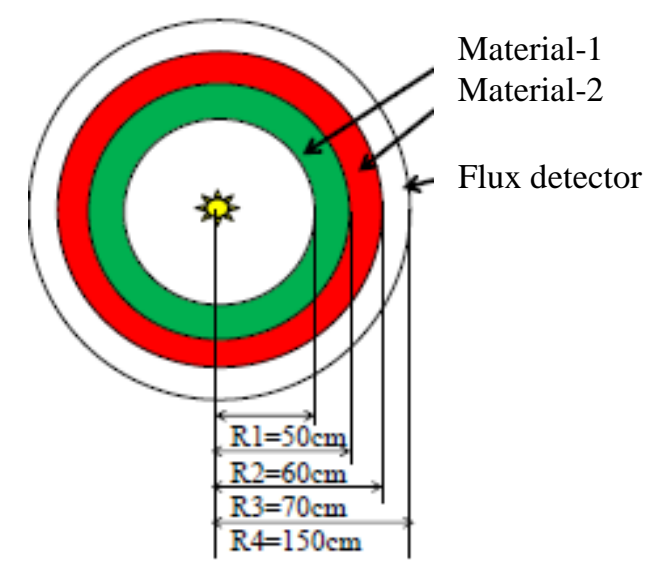

Figure 2. Schematic representation of the Monte Carlo model

Table 2. Geometric conditions of the calculations

\begin{tabular}{ccc}
\hline Calculation & Material-1(Inside) & Material-2(Outside) \\
\hline 1 & Double Shield & Double-Shield \\
2 & $\mathrm{~Pb}$ & $\mathrm{H}_{3} \mathrm{BO}_{3}$ \\
3 & $\mathrm{H}_{3} \mathrm{BO}_{3}$ & $\mathrm{~Pb}$ \\
\hline
\end{tabular}

\section{Results and discussion}

\subsection{Neutron transmission rate}

The TOF spectra obtained by measuring transmission neutrons are shown in Figure 3. The TOF spectrum without sample (Blank), which means an incident neutron spectrum on the sample, is represented by black 
open circle in Fig. 3. The neutron attenuation rate was obtained for each energy region by comparing with the incident neutron flux as shown in Figure 4. Below $1 \mathrm{eV}$, there are no differences between Double Shield and polyethylene mixed with $10 \%$ boron oxide. In the epithermal region, the attenuation rate of Double Shield with a thickness of $5 \mathrm{~cm}$ was better than that of $\mathrm{Pb}$ with a thickness of $10 \mathrm{~cm}$.

\subsection{Dose rate of leakage neutron and gamma rays}

Figures 5 and $\mathbf{6}$ show the comparison of leakage neutron and gamma-ray dose rate changes among shielding materials. The gamma-ray dose rate is about $10 \%$ of the neutron dose rate without the shielding material. It is worth noting that the mixed radiation field used in the experiment reproduces a BNCT irradiation field in the viewpoints of balance between neutron and gamma-ray doses.

The dose rate of leakage neutron for Double Shield was converging on the background level at the thickness of $30 \mathrm{~cm}$. The thickness is about 1.5 times that of the polyethylene mixed with $10 \%$ boron oxide. The dose rate of leakage gamma ray for Double Shield was decreasing with thickness exponentially. On the other

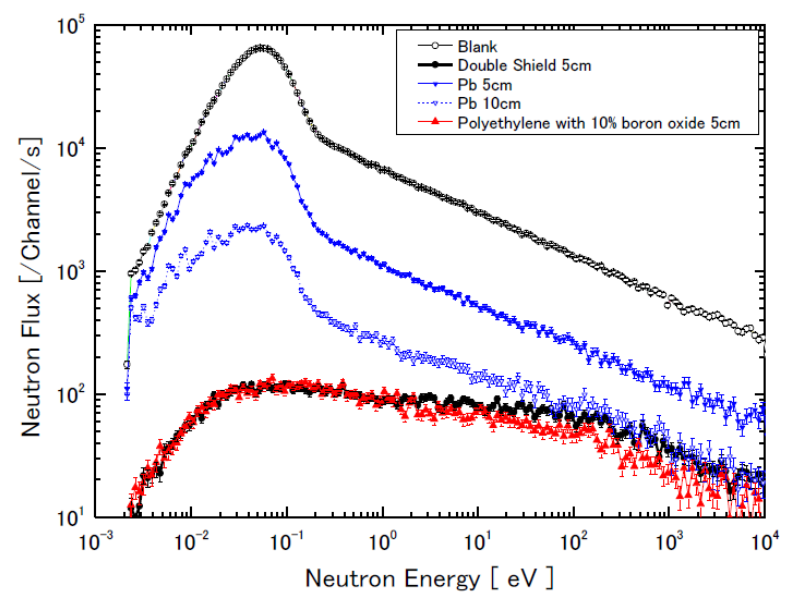

Figure 3. Transmission neutron TOF spectra for shielding materials

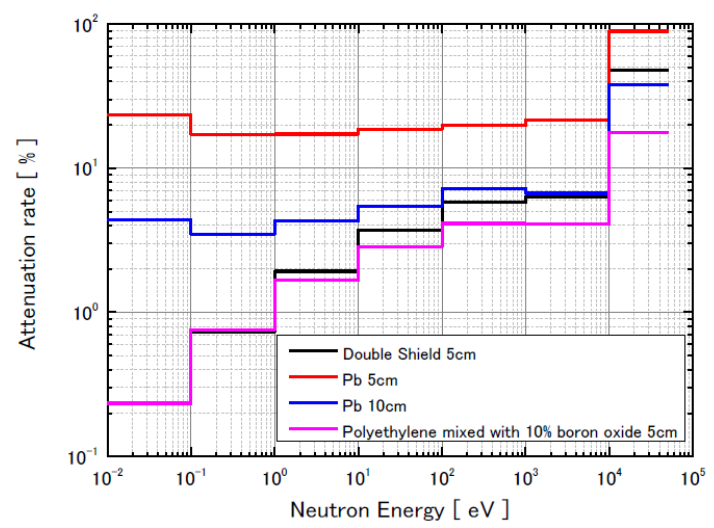

Figure 4. Comparison of neutron attenuation rate among shielding materials hands, the dose rate for polyethylene mixed with $10 \%$ boron oxide was converging on the level higher than background around the thickness of $25 \mathrm{~cm}$. It seems that primary and secondary gamma rays cannot be reduced. The comparison of leakage total dose rate changes is shown in Figure 7. It was found that the total dose rate of Double Shield was effectively decreasing compared with $\mathrm{Pb}$ and polyethylene mixed with $10 \%$ boron oxide.

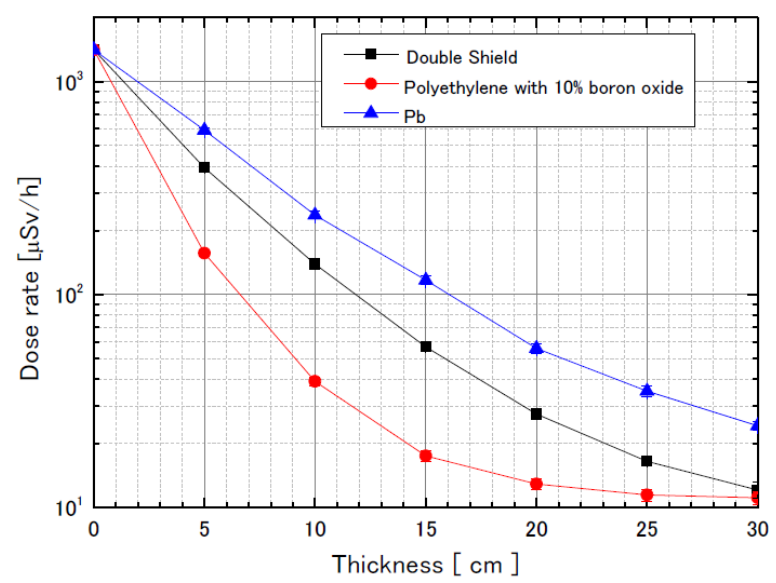

Figure 5. Comparison of leakage neutron dose rate changes among shielding materials

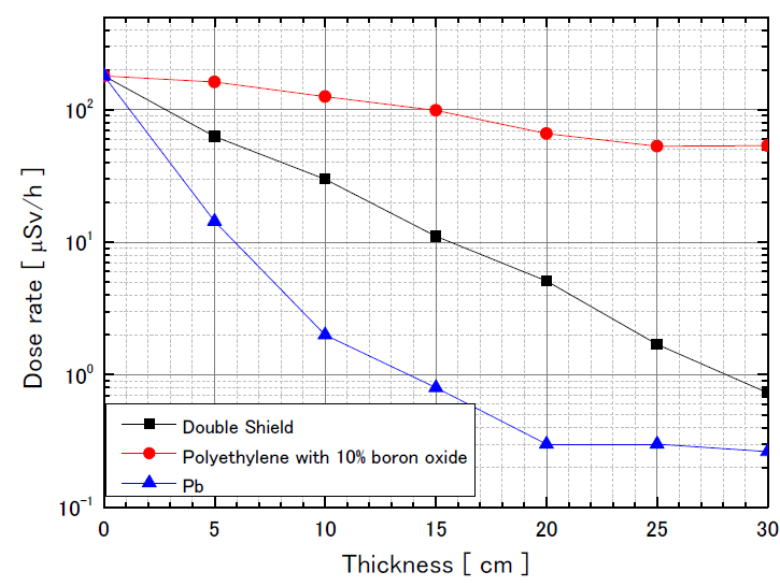

Figure 6. Comparison of leakage gamma-ray dose rate changes among shielding materials

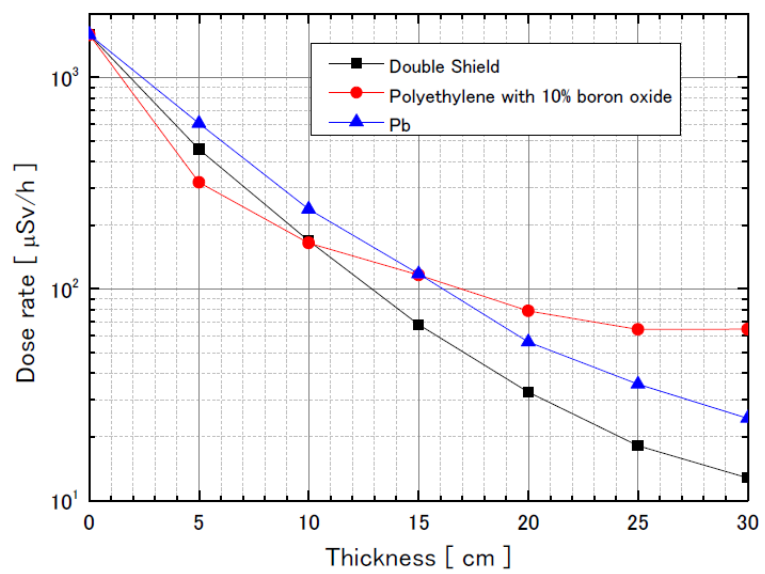

Figure 7. Comparison of leakage total dose rate changes among shielding materials 


\subsection{Calculation results}

The comparison of neutron energy spectra obtained by calculation-1, 2 and 3 is shown in Figure 8. It is found that the high energy components above $100 \mathrm{keV}$ are effectively reduced by locating lead inside. The results show that the inelastic scattering by lead has an important role to reduce the high energy components. In the case of calculation-1, the reduction rate for high energy neutron is close to that of calculation-2. In the case of calculation-3, the reduction rates in the high energy region ( $>100 \mathrm{keV}$ ) and low energy region $(<0.1$ $\mathrm{eV})$ are much smaller than those of the other cases since the slowing down is not sufficiently done in the material-1 region. Therefore, it is expected that the high-energy neutron can be effectively reduced by mixing $\mathrm{Pb}$-shots to the shielding material for the neutron source with a Maxwell fission spectrum.

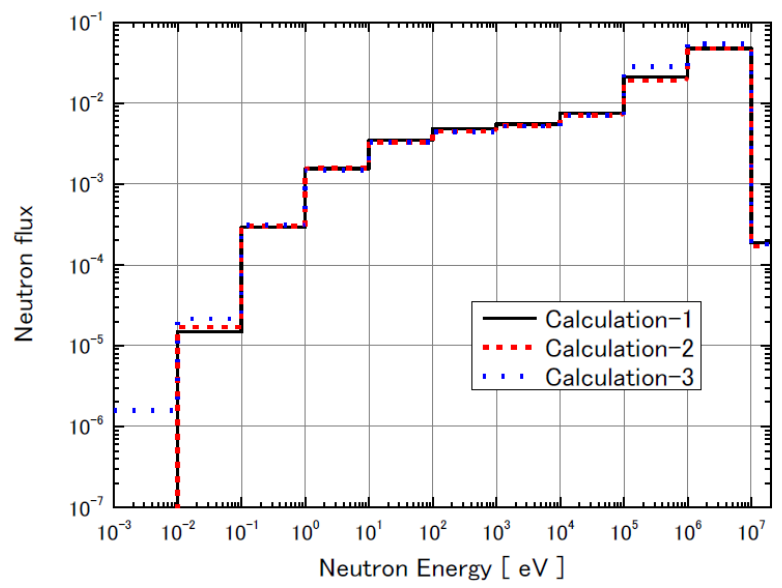

Figure 8. Comparison of neutron energy spectra obtained by calculation-1,2 and 3

\section{Summary}

We have carried out the transmission neutron measurement with the TOF technique and obtained the attenuation rate of the new shielding material called as "Double Shield" for each energy region. Moreover, the leakage neutron and gamma-ray dose rates were measured for several shielding materials. Based on the results of those experiments, it was verified that Double Shield has a higher shielding performance compared to usual shielding materials in the neutrons and gamma-rays mixed radiation fields.

\section{Acknowledgements}

The authors would like to express their thanks to the linac staff of the Research Reactor Institute, Kyoto University (KURRI) for making it possible to operate the accelerator steadily.

\section{References}

[1] J. Ghassoun, B. Chkillou and A. Jehouani, Spatial and spectral characteristics of a compact system neutron beam designed for BNCT facility, Applied Radiation and Isotopes, 67 (2009), pp. 560-564.

[2] H. Tanaka, Y. Sakurai, M. Suzuki, S. Masunaga, Y. Kinashi, G. Kashino, Y. Liu, T. Mitsumoto, S. Yajima, H. Tsutsui, A. Maruhashi and K. Ono, Characteristics comparison between a cyclotron-based neutron source and KUR-HWNIF for boron neutron capture therapy, Nucl. Instrum. Meth., B 267 (2009), pp.1970-1977.

[3] K. Kobayashi, G. Jin, S. Yamamoto, K. Takami, Y. Kimura, T. Kozuka and Y. Fujita, KURRI-Linac as a neutron source for irradiation, Annu. Rep. Res. Reactor Inst. Kyoto Univ., 22 (1989) 142-153.

[4] J. F. Briesmeister, MCNP-A General Monte Carlo $N$-Particle Transport Code Version 4C, Los Alamos, NM: Los Alamos National Laboratory, (2000). 